\title{
Time and Norm Optimality of Weakly Singular Controls
}

\author{
H.O. Fattorini
}

\begin{abstract}
Let $\bar{u}(t)$ be a control that satisfies the infinite-dimensional version of Pontryagin's maximum principle for a linear control system, and let $z(t)$ be the costate associated with $\bar{u}(t)$. It is known that integrability of $\|z(t)\|$ in the control interval $[0, T]$ guarantees that $\bar{u}(t)$ is time and norm optimal. However, there are examples where optimality holds (or does not hold) when $\|z(t)\|$ is not integrable. This paper presents examples of both cases for a particular semigroup (the right translation semigroup in $L^{2}(0, \infty)$ ).
\end{abstract}

Mathematics Subject Classification (2000). 93E20, 93E25.

Keywords. Linear control systems in Banach spaces, norm optimal problem, time optimal problem, weakly singular controls, costate.

\section{Introduction}

We consider two optimal control problems for the system

$$
y^{\prime}(t)=A y(t)+u(t), \quad y(0)=\zeta
$$

with controls $u(\cdot) \in L^{\infty}(0, T ; E)$, where $A$ generates a strongly continuous semigroup $S(t)$ in a Banach space $E$. The first is the norm optimal problem, where we drive the initial point $\zeta$ to a point target,

$$
y(T)=\bar{y}
$$

in a fixed time interval $0 \leq t \leq T$ minimizing $\|u(\cdot)\|_{L^{\infty}(0, T ; E)}$. The second is the time optimal problem, where we drive to the target with a bound on the norm of the control (say $\|u(\cdot)\|_{L^{\infty}(0, T ; E)} \leq 1$ ) in optimal time $T$. The solution or trajectory of (1.1) is the continuous function

$$
y(t)=y(t, \zeta, u)=S(t) \zeta+\int_{0}^{t} S(t-\sigma) u(\sigma) d \sigma .
$$


For the time optimal problem, controls in $L^{\infty}(0, T ; E)$ with $\|u(\cdot)\|_{L^{\infty}(0, T ; E)} \leq 1$ are named admissible.

Necessary and sufficient conditions for norm and time optimality can be given in terms of the maximum principle (1.5) below which requires the construction of spaces of multipliers (final values of costates). We summarize this construction from [4] or [5], Section 2.3. When $A$ has a bounded inverse, we define the space $E_{-1}^{*}$ as the completion of $E^{*}$ in the norm

$$
\left\|y^{*}\right\|_{E_{-1}^{*}}=\left\|\left(A^{-1}\right)^{*} y^{*}\right\|_{E^{*}}
$$

Each $S(t)^{*}$ can be extended to an operator $S(t)^{*}: E_{-1}^{*} \rightarrow E_{-1}^{*}$, and $Z_{w}(T) \subseteq E_{-1}^{*}$ consists of all $z \in E_{-1}^{*}$ such that $S(t)^{*} z \in E^{*}$ and $^{1}$

$$
\|z\|_{Z_{w}(T)}=\int_{0}^{T}\left\|S(t)^{*} z\right\|_{E^{*}} d t<\infty .
$$

The space $Z_{w}(T)$ equipped with $\|\cdot\|_{Z_{w}(T)}$ is a Banach space. All spaces $Z_{w}(T)$ coincide (that is, $Z_{\omega}(T)=Z_{\omega}\left(T^{\prime}\right)$ for any $T, T^{\prime}>0$ and the norms $\|\cdot\|_{Z_{w}(T)}$, $\|\cdot\|_{Z_{w}\left(T^{\prime}\right)}$ are equivalent). $Z_{w}(T)$ is an example of a multiplier space, an arbitrary linear space $\mathcal{Z} \supseteq E^{*}$ to which $S(t)^{*}$ can be extended in such a way that $S(t)^{*} \mathcal{Z} \subseteq$ $E^{*}$ for $t>0$. When $A$ does not have a bounded inverse, the construction of the spaces is modified as follows. Since $A$ is a semigroup generator, $(\lambda I-A)^{-1}$ exists for $\lambda>\omega$ and $E_{-1}^{*}$ is the completion of $E^{*}$ in any of the equivalent norms

$$
\left\|y^{*}\right\|_{E_{-1}^{*}, \lambda}=\left\|\left((\lambda I-A)^{-1}\right)^{*} y^{*}\right\|_{E^{*}}, \quad(\lambda>\omega) .
$$

The definition of $Z_{w}(T)$ (and of multiplier spaces) is the same. See [5], Section 2.3 for proofs of these results and additional details.

A control $\bar{u}(\cdot) \in L^{\infty}(0, T ; E)$ satisfies Pontryagin's maximum principle if

$$
\left\langle S(T-t)^{*} z, \bar{u}(t)\right\rangle=\max _{\|u\| \leq \rho}\left\langle S(T-t)^{*} z, u\right\rangle \quad \text { a.e. in } 0 \leq t<T,
$$

where $\langle\cdot, \cdot\rangle$ is the duality of the space $E$ and the dual $E^{*}$, with $\rho=\|\bar{u}(\cdot)\|_{L^{\infty}(0, T ; E)}$ and $z$ in some multiplier space $\mathcal{Z}$. We call $z$ the multiplier and $z(t)=S(T-t)^{*} z$ the costate corresponding to (or associated with) the control $\bar{u}(t)$. We assume that (1.5) is nontrivial; this means $S(T-t)^{*} z$ is not identically zero in the interval $0 \leq t<T$, although it may be zero in part of the interval (in which part (1.5) says nothing about $\bar{u}(t))$. That (1.5) is nontrivial implies that $z \neq 0$. The maximum principle is especially simple when $E$ is a Hilbert space; it reduces to

$$
\bar{u}(t)=\rho \frac{S(T-t)^{*} z}{\left\|S(T-t)^{*} z\right\|} \quad(T-\delta<\sigma \leq T),
$$

where $0 \leq t<\delta$ is the maximal interval where $S(t)^{*} z \neq 0$; if $\delta \geq T$ the interval is $0<\sigma \leq T$.

\footnotetext{
${ }^{1}$ Without further assumptions, the semigroup $S(t)^{*}$ may not be strongly continuous, or even strongly measurable (consider, for instance, the translation semigroup $S(t) y(x)=y(x-t)$ in $\left.E=L^{1}(\infty, \infty)\right)$. However, $S(t)^{*}$ is always $E$-weakly continuous, which guarantees that $\left\|S(t)^{*}\right\|$ is lower semicontinuous, hence measurable. This justifies the integral (1.4).
} 
A large part of the theory of optimal controls for the system (1.1) deals with the relation between optimality and the maximum principle (1.5). All one has (at present) are separate necessary and sufficient conditions for optimality based on the maximum principle (Theorem 1.1 below). We call an optimal control $\bar{u}(t)$ regular if it satisfies (1.5) with $z \in Z_{w}(T)$.

Theorem 1.1. Assume $\bar{u}(t)$ drives $\zeta \in E$ to $\bar{y}=y(T, \zeta, \bar{u})$ time or norm optimally in the interval $0 \leq t \leq T$ and that

$$
\bar{y}-S(T) \zeta \in D(A) .
$$

Then $\bar{u}(t)$ is regular. Conversely, let $\bar{u}(t)$ be a regular control. Then $\bar{u}(t)$ drives $\zeta \in E$ to $\bar{y}=y(T, \zeta, \bar{u})$ norm optimally in the interval $0 \leq t \leq T$; if $\rho=1$ the drive is time optimal.

For the proof see [4], Theorem 5.1, [5], Theorem 2.5.1; we note that in the sufficiency half of Theorem 1.1 no conditions of the type of (1.7) are put on the initial value $\zeta$ or the target $\bar{y}^{2}$

Following the terminology in [5] we call a control weakly singular if it satisfies the maximum principle (1.5) but the costate does not satisfy the integrability condition (1.4) (that is, $z \notin Z_{w}(T)$ ). The following question arises: is a weakly singular control (norm, time) optimal? The answer to this question is "not necessarily" and examples of weakly singular controls that are (or are not) optimal are known. It is proved in [2] (see [5], Section 3.4) that for the (self-adjoint) multiplication operator

$$
\mathcal{A} u(\lambda)=-\lambda u(\lambda)
$$

in $L^{2}(0, \infty)$, which generates the analytic semigroup

$$
\mathcal{S}(t) u(\lambda)=e^{-\lambda t} u(t)
$$

there exist optimal controls for (1.1) satisfying the maximum principle (1.5) where the growth of the costate $z(t)$ as $t$ approaches the final time $T$ is $\approx C /(T-t)$ in the sense that

$$
(T-t)\|z(t)\|_{E^{*}}=(T-t)\left\|S(T-t)^{*} z\right\|_{E^{*}} \rightarrow C \quad \text { as } t \rightarrow T
$$

with $0<C<\infty$. These controls cannot satisfy (1.4), thus they are weakly singular. On the other hand there exist controls satisfying (1.5) and

$$
(T-t)^{\alpha}\|z(t)\|_{E^{*}}=(T-t)^{\alpha}\left\|S(T-t)^{*} z\right\|_{E^{*}} \rightarrow C \quad \text { as } t \rightarrow T
$$

with $\alpha>1$ and $0<C<\infty$ (thus weakly singular) that are not time or norm optimal. We provide in this paper similar examples for the right translation semigroup

\footnotetext{
${ }^{2}$ The statement on time optimality, however, needs additional assumptions on the initial condition $\zeta$ and the target $\bar{y}$. These conditions are satisfied if either $\zeta=0$ or $\bar{y}=0$ [5], Theorem 2.5.7. We point out that the conditions are on the "size" of $\zeta \bar{y}$, not on their smoothness like (1.7); for instance, for $\zeta=0, \bar{y}$ may be an arbitrary element of $E$. We also need to assume that $S(t)^{*} z \neq 0$ in the entire interval $0 \leq t \leq T$.
} 
$S(t)$ in $L^{2}(0, \infty)$ defined by

$$
S(t) y(x)= \begin{cases}y(x-t) & (x \geq t) \\ 0 & (x<t) .\end{cases}
$$

Although the technical means are totally different, the examples are of the same sort as those in [2]; there are controls that satisfy (1.9) and are optimal, whereas there are controls with faster increase of $\|z(t)\|$ which are not optimal. What is remarkable about the examples in this paper is that they resemble similar examples for semigroups as different as (1.8), analytic with (1.1) an abstract parabolic equation. The semigroup (1.11) under study here is isometric, with associated equation (1.1) having a finite velocity of propagation, thus qualifying as "abstract hyperbolic".

On the basis of this similar behavior of controls for very different semigroups it is tempting to guess that there must exist some sort of classification of weakly singular controls (as optimal or nonoptimal) which is based on the growth of the norm of the costate $z(t)=S(T-t)^{*} z$ as $t \rightarrow T$ and holds for arbitrary semigroups. There seems to be no such general result except [3] Lemma 8.3, [5] Lemma 3.5.9 where the generator is self-adjoint in Hilbert space and $\left\|S(t)^{*} z\right\|$ has "hyperpower growth" as $t \rightarrow 0$; this means

$$
\int_{0}^{1} \frac{\left\|S(r \sigma)^{*} z\right\|}{\sigma\left\|S(\sigma)^{*} z\right\|} d \sigma<\infty
$$

for some $r$ in the range $1<r<2$ (the adjoint may be omitted since the semigroup $S(t)$ is self-adjoint). Under (1.12), the control corresponding to the multiplier $z$ is not optimal. Condition (1.12) cannot hold if (1.10) is satisfied for any $\alpha>0$; in fact, in this case

$$
\frac{\left\|S(r \sigma)^{*} z\right\|}{\sigma\left\|S(\sigma)^{*} z\right\|} \approx \frac{C \sigma^{\alpha}}{\sigma C(r \sigma)^{\alpha}}=\frac{1}{r^{\alpha} \sigma}
$$

making the integral infinite. However, there is a wide gap between hyperpower growth and power growth like (1.9), and nothing is known for intermediate growths.

We mention in passing the results on multipliers in [6]. When the semigroup satisfies

$$
S(t) E=E \quad(t>0)
$$

then every multiplier space satisfies $\mathcal{Z}=Z_{w}(T)=E^{*}$, that is, all multipliers in (1.5) automatically belong to $E^{*}$; this makes moot the question of the growth of $\|z(t)\|$ as $t \rightarrow T$. It is also shown in [6] that (under the assumption that $E$ is reflexive and separable) (1.13) is a necessary condition for all multipliers to belong to $E^{*}$. Moreover, condition (1.7) can be dropped from Theorem 1.1 in case (1.12) holds: all time or norm optimal controls satisfy (1.5) with a multiplier $z \in E^{*}$.

\section{The right translation semigroup}

The space is $E=L^{2}(0, \infty)$. Its elements $y(x)$ (defined in $x \geq 0$ ) are extended as $y(x)=0$ for $x<0$. The right translation semigroup $S(t)$ defined by (1.11) is 
strongly continuous and isometric in $L^{2}(0, \infty)$. The adjoint semigroup is the left translation (and chop-off) semigroup

$$
S(t)^{*} y(x)= \begin{cases}y(x+t) & (x \geq 0) \\ 0 & (x<0)\end{cases}
$$

We have

$$
S(t)^{*} S(t)=I, \quad S(t) S(t)^{*} y(x)=\chi_{t}(x) y(x)
$$

where $\chi_{t}(x)$ is the characteristic function of $[t, \infty)$. The infinitesimal generator $A$ of $S(t)$ is

$$
A y(x)=-y^{\prime}(x)
$$

with domain $D(A)=\left\{\right.$ all $y(\cdot) \in L^{2}(0, \infty)$ with $y^{\prime}(\cdot)$ in $L^{2}(0, \infty)$ and $\left.y(0)=0\right\}$, the derivative understood in the sense of distributions. The semigroup $S(t)$ is associated with the control system

$$
\begin{aligned}
\frac{\partial y(t, x)}{\partial t} & =-\frac{\partial y(t, x)}{\partial x}+u(t, x) \\
y(0, x) & =\zeta(x), \quad y(t, 0)=0,
\end{aligned}
$$

in the sense that $S(t)$ is the propagation semigroup of the homogeneous equation $(u(t, x)=0)$. Formula (1.3) for the control $u(t)(x)=u(t, x)$ is

$$
\begin{aligned}
y(t, x, \zeta, u) & =y(t, \zeta, u)(x)=\left(S(t) \zeta+\int_{0}^{t} S(t-\sigma) u(\sigma) d \sigma\right)(x) \\
& =\zeta(x-t)+\int_{0}^{t} u(\sigma, x-(t-\sigma)) d \sigma
\end{aligned}
$$

thus the contribution of $u(\sigma, x)$ to $y(t, x, \zeta, u)$ is the integral of $u(\sigma, x)$ over the intersection with the positive quadrant of the characteristic line $(\sigma, x-(t-\sigma))$ joining $(0, x-t)$ with $(t, x)$, as shown in Figure 1 .

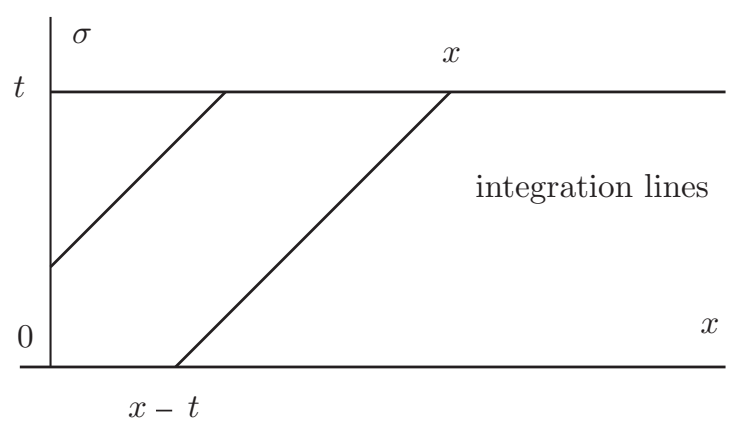

FiguRe 1 
We name $\mathcal{Z}$ the space of all measurable $z(x)$ defined in $x>0$ and such that

$$
\kappa(\sigma, z)=\left\|S(\sigma)^{*} z(\cdot)\right\|=\sqrt{\int_{0}^{\infty} z(x+\sigma)^{2} d x}=\sqrt{\int_{\sigma}^{\infty} z(x)^{2} d x}<\infty
$$

in $\sigma>0$. The space $^{3} Z(T)$ consists of all $z(\cdot) \in \mathcal{Z}$ with

$$
\int_{0}^{T}\left\|S(\sigma)^{*} z(\cdot)\right\| d \sigma=\int_{0}^{T} \kappa(\sigma, z) d \sigma<\infty .
$$

Since we are in a Hilbert space (1.6) applies and any control that satisfies the maximum principle (1.5) is given a.e. by

$$
\bar{u}(\sigma, x)=\rho \frac{S(T-\sigma)^{*} z(x)}{\left\|S(T-\sigma)^{*} z(\cdot)\right\|}=\rho \chi_{0}(x) \frac{z(x+(T-\sigma))}{\kappa(T-\sigma, z)} \quad(T-\delta<\sigma \leq T),
$$

where $0 \leq t<\delta$ is the maximal interval where $S(t)^{*} z \neq 0$; if $\delta \geq T$, the interval in $(2.7)$ is $0<\sigma \leq T$.

Using the second equality (2.2) and assuming for simplicity that $\rho=1$ we obtain

$$
S(T-\sigma) \bar{u}(\sigma)(x)=\frac{S(T-\sigma) S(T-\sigma)^{*} z(x)}{\left\|S(T-\sigma)^{*} z(\cdot)\right\|}=\frac{\chi_{T-\sigma}(x) z(x)}{\kappa(T-\sigma, z)} \quad(0 \leq \sigma<T)
$$

whenever $\kappa(T-\sigma, z) \neq 0$. Formula (2.5) for $t=T$ becomes

$$
\begin{aligned}
& y(T, x, \zeta, \bar{u})(x)=S(T) \zeta(x)+\int_{0}^{T} S(T-\sigma) \bar{u}(\sigma, x) d \sigma \\
& =\zeta(x-T)+\int_{0}^{T} \frac{\chi_{T-\sigma}(x) z(x)}{\kappa(T-\sigma, z)} d \sigma=\zeta(x-T)+z(x) \int_{0}^{T} \frac{\chi_{T-\sigma}(x)}{\kappa(T-\sigma, z)} d \sigma \\
& =\zeta(x-T)+z(x) \int_{0}^{T} \frac{\chi_{\sigma}(x)}{\kappa(\sigma, z)} d \sigma=\zeta(x-T)+z(x) \omega(T, x, z),
\end{aligned}
$$

where

$$
\omega(T, x, z)=\int_{0}^{T} \frac{\chi_{\sigma}(x)}{\kappa(\sigma, z)} d \sigma=\int_{0}^{\min (x, T)} \frac{d \sigma}{\kappa(\sigma, z)} .
$$

If we drive from 0 to $\bar{y}(x)$ in time $T$, the target $\bar{y}(x)$ and the costate $z(x)$ are related by

$$
\bar{y}(x)=y(T, x, 0, \bar{u})=z(x) \omega(T, x, z),
$$

so that the target $\bar{y}(x)$ is a multiple of the multiplier $z(x)$ in $x \geq T$.

${ }^{3}$ We drop the subindex $w$ since $S(t)^{*}$ is strongly continuous. 


\section{Weakly singular controls, I}

We use the family of multipliers

$$
z(x)=\frac{1}{x^{\alpha}} \quad\left(\alpha>\frac{1}{2}\right)
$$

associated with the controls

$$
\bar{u}_{\alpha}(\sigma, x)=\frac{\chi_{0}(x)}{\kappa(T-\sigma, z)(x+(T-\sigma))^{\alpha}} \quad(0 \leq \sigma \leq T) .
$$

We have

$$
\kappa(\sigma, z)^{2}=\int_{\sigma}^{\infty} \frac{d x}{x^{2 \alpha}}=\frac{\sigma^{1-2 \alpha}}{2 \alpha-1}=\frac{1}{(2 \alpha-1) \sigma^{2 \alpha-1}}
$$

thus

$$
\kappa(\sigma, z)=\frac{1}{\sqrt{2 \alpha-1} \sigma^{\alpha-1 / 2}}
$$

and, in view of $(2.6)$ the control $\bar{u}_{\alpha}(\sigma, x)$ is regular $(z(\cdot) \in Z(T))$ if and only if

$$
\alpha-\frac{1}{2}<1 \quad \Longleftrightarrow \quad \alpha<\frac{3}{2}
$$

on the other hand $z(\cdot) \in \mathcal{Z}$ (thus $\bar{u}_{\alpha}(\sigma, x)$ is weakly singular) for arbitrary $\alpha \geq 3 / 2$. Combining (3.2) and (3.3)

$$
\bar{u}_{\alpha}(\sigma, x)=\frac{\sqrt{2 \alpha-1} \chi_{0}(x)(T-\sigma)^{\alpha-1 / 2}}{(x+(T-\sigma))^{\alpha}} \quad(0 \leq \sigma \leq T) .
$$

We have

$$
\begin{aligned}
& \int_{\sigma}^{\infty} \bar{u}_{\alpha}(\sigma, x)^{2} d x=(2 \alpha-1)(T-\sigma)^{2 \alpha-1} \int_{\sigma}^{\infty}(x+(T-\sigma))^{-2 \alpha} d x \\
& =\left.(2 \alpha-1)(T-\sigma)^{2 \alpha-1} \frac{(x+(T-\sigma))^{1-2 \alpha}}{1-2 \alpha}\right|_{x=\sigma} ^{x=\infty}=\left(\frac{T-\sigma}{T}\right)^{2 \alpha-1}, \\
& \int_{0}^{\sigma} \bar{u}_{\alpha}(\sigma, x)^{2} d x=(2 \alpha-1)(T-\sigma)^{2 \alpha-1} \int_{0}^{\sigma}(x+(T-\sigma))^{-2 \alpha} d x \\
& =\left.(2 \alpha-1)(T-\sigma)^{2 \alpha-1} \frac{(x+(T-\sigma))^{1-2 \alpha}}{1-2 \alpha}\right|_{x=0} ^{x=\sigma}=1-\left(\frac{T-\sigma}{T}\right)^{2 \alpha-1} .
\end{aligned}
$$

Since $2 \alpha-1>0$ we have the proof of

Lemma 3.1. Let $0 \leq \sigma \leq T$. Let

$$
I(\alpha)=\int_{0}^{\sigma} \bar{u}_{\alpha}(\sigma, x)^{2} d x, \quad J(\alpha)=\int_{\sigma}^{\infty} \bar{u}_{\alpha}(\sigma, x)^{2} d x \quad\left(\alpha>\frac{1}{2}\right) .
$$

Then $I(\alpha)$ (resp. $J(\alpha))$ is a decreasing (resp. increasing) function of $\alpha$.

From (2.10) and (3.3) we have

$$
\omega(T, x, z)=\sqrt{2 \alpha-1} \int_{0}^{x} \sigma^{\alpha-1 / 2} d \sigma=\frac{\sqrt{2 \alpha-1}}{\alpha+1 / 2} x^{\alpha+1 / 2}
$$


for $x \leq T$; for $x \geq T$

$$
\omega(T, x, z)=\sqrt{2 \alpha-1} \int_{0}^{T} \sigma^{\alpha-1 / 2} d \sigma=\frac{\sqrt{2 \alpha-1}}{\alpha+1 / 2} T^{\alpha+1 / 2} .
$$

Formula (2.11) implies that the control $\bar{u}_{\alpha}(\sigma, x)$ in (3.4) drives 0 to the target

$$
\begin{array}{ll}
\bar{y}(x)=\frac{\sqrt{2 \alpha-1}}{\alpha+1 / 2} x^{1 / 2} & (x \leq T), \\
\bar{y}(x) & =\frac{\sqrt{2 \alpha-1}}{\alpha+1 / 2} \frac{T^{\alpha+1 / 2}}{x^{\alpha}} \quad(x>T) .
\end{array}
$$

Figure 2 shows $\bar{y}_{3 / 2}(x)$ for $T=1$.

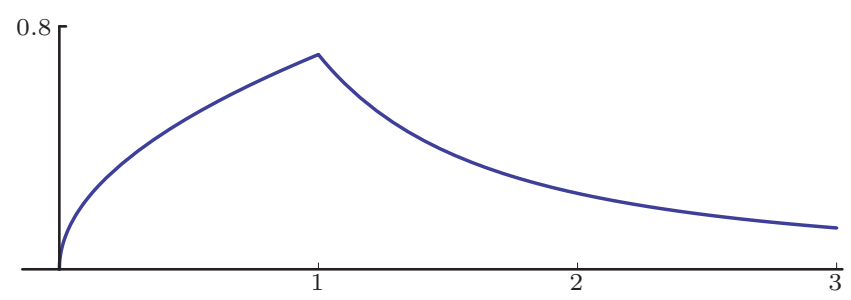

FiguRE 2

As a consequence of Theorem 1.1 we obtain

Theorem 3.2. If $\alpha<3 / 2$ the control $\bar{u}_{\alpha}(\sigma, x)$ is regular, thus it drives 0 to $\bar{y}_{\alpha}(x)$ time and norm optimally.

We prove below that this is no longer true if $\alpha>3 / 2$. The proof is based on the fact that the function

$$
K(\alpha)=\frac{\sqrt{2 \alpha-1}}{\alpha+1 / 2}
$$

(the factor in (3.7)) has the unique maximum $3 / 2$ in $\alpha \geq 1 / 2($ with $K(3 / 2)=$ $1 / \sqrt{2})$. The graph of $K(\alpha)$ is shown in Figure 3.

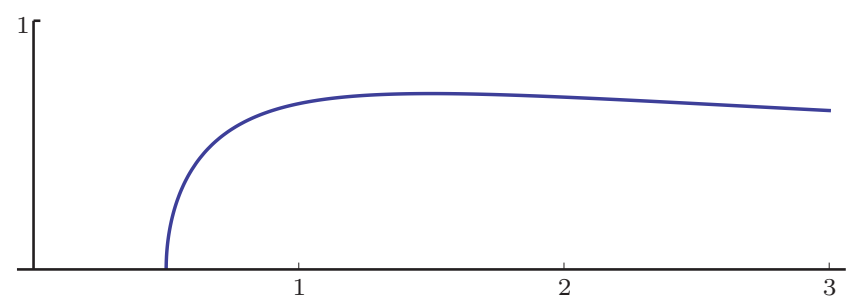

FiguRe 3 
Let $\alpha>3 / 2$, so that $K(3 / 2)>K(\alpha)$. We improve the norm-performance provided by the control $\bar{u}_{\alpha}(\sigma, x)$ constructing a control $\tilde{u}_{\alpha}(\sigma, x)$ "by pieces" as follows (see Figure 4).

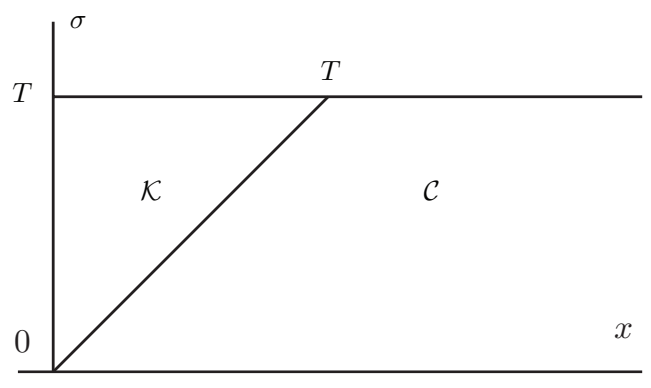

Figure 4

In the triangle $\mathcal{K}$ we define

$$
\tilde{u}_{\alpha}(\sigma, x)=\frac{K(\alpha)}{K(3 / 2)} \bar{u}_{3 / 2}(\sigma, x) .
$$

The integration formula (2.5) (see also Figure 1) shows that this new definition affects the target $\bar{y}(x)$ only in the interval $0 \leq t \leq T$. There is actually no change in the target, since in this interval the target hit by $\tilde{u}_{\alpha}(\sigma, x)$ is

$$
\bar{y}(x)=\frac{K(\alpha)}{K(3 / 2)} \bar{y}_{3 / 2}(x)=\bar{y}_{\alpha}(x) \text {. }
$$

Using the first part of Lemma 3.1 we obtain

$$
\int_{0}^{\sigma} \tilde{u}_{\alpha}(\sigma, x)^{2} d x=\frac{K(\alpha)}{K(3 / 2)} \int_{0}^{\sigma} \bar{u}_{3 / 2}(\sigma, x)^{2} d x \leq \frac{K(\alpha)}{K(3 / 2)} \int_{0}^{\sigma} \bar{u}_{\alpha}(\sigma, x)^{2} d x .
$$

As a first approximation we don't modify the control $\bar{u}_{\alpha}(\sigma, x)$ in the complement $\mathcal{C}$ of $\mathcal{K}$, so that we drive to the target $\bar{y}_{\alpha}(x)$ with

$$
v(\sigma, x)= \begin{cases}\tilde{u}_{\alpha}(\sigma, x) & (\sigma, x) \in \mathcal{K} \\ \bar{u}_{\alpha}(\sigma, x) & (\sigma, x) \in \mathcal{C} .\end{cases}
$$

Using (3.5), (3.6) and (3.9),

$$
\begin{aligned}
\|v(\sigma, \cdot)\|_{L^{2}(0, \infty)} & =\int_{0}^{\infty} v(\sigma, x)^{2} d x \\
& =\int_{0}^{\sigma} v(\sigma, x)^{2} d x+\int_{\sigma}^{\infty} v(\sigma, x)^{2} d x \\
& \leq \frac{K(\alpha)}{K(3 / 2)} \int_{0}^{\sigma} \bar{u}_{\alpha}(\sigma, x)^{2} d x+\int_{\sigma}^{\infty} \bar{u}_{\alpha}(\sigma, x)^{2} d x \\
& =\frac{K(\alpha)}{K(3 / 2)}\left(1-\left(\frac{T-\sigma}{T}\right)^{2 \alpha-1}\right)+\left(\frac{T-\sigma}{T}\right)^{2 \alpha-1}=\eta(\sigma)
\end{aligned}
$$


The fact that $\eta(\alpha)<1$ in $0<\sigma \leq T$ implies right away that $v(\cdot, \cdot)$ (thus $\bar{u}_{\alpha}(\cdot, \cdot)$ ) is not time optimal, since the bang-bang theorem [1] Theorem 2.2, [5] Theorem 2.1.3 says that time optimal controls $\bar{u}(\sigma)$ for $(1.1)$ must satisfy $\|\bar{u}(\sigma)\|=1$ almost everywhere. However, $\eta(\sigma)<1$ does not imply that $v_{\alpha}(\cdot, \cdot)$ is not norm optimal since $\eta(0)=1$ (time optimal $\rightarrow$ norm optimal but the converse is not true). Thus, we must prove directly that $\bar{u}_{\alpha}(\cdot, \cdot)$ is not norm optimal, which requires modification of $v(\cdot, \cdot)$ in $\mathcal{C}$. This will be done by further subdivision of $\mathcal{C}$ into two regions $\mathcal{C}_{1}$ and $\mathcal{C}_{2}$ indicated in Figure 5; the parameter $N>0$ will be determined later.

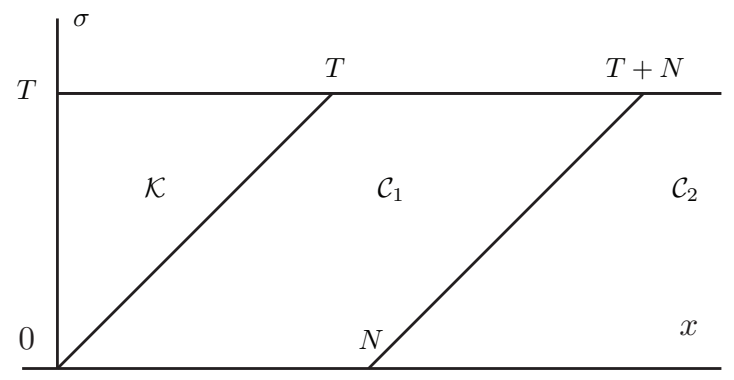

FigURE 5

A computation entirely similar to (3.6) shows

$$
\int_{\sigma}^{\sigma+N} \bar{u}_{\alpha}(\sigma, x)^{2} d x=\left(\frac{T-\sigma}{T}\right)^{2 \alpha-1}-\left(\frac{T-\sigma}{T+N}\right)^{2 \alpha-1} .
$$

We introduce the control

$$
\bar{v}_{\alpha}(\sigma, x)=\frac{\sqrt{2 \alpha-1} \chi_{0}(x) \sigma^{\alpha-1 / 2}}{(x+(T-\sigma))^{\alpha}} \quad(0 \leq \sigma \leq T) .
$$

After another computation like (3.6),

$$
\int_{\sigma+N}^{\infty} \bar{v}_{\alpha}(\sigma, x)^{2} d x=\left(\frac{\sigma}{T+N}\right)^{2 \alpha-1} .
$$

Moreover,

Since

$$
\bar{v}_{\alpha}(\sigma, x-(T-\sigma))=\frac{\sqrt{2 \alpha-1} \chi_{0}(x) \sigma^{\alpha-1 / 2}}{x^{\alpha}} .
$$

$$
\bar{u}_{\alpha}(\sigma, x-(T-\sigma))=\frac{\sqrt{2 \alpha-1} \chi_{0}(x)(T-\sigma)^{\alpha-1 / 2}}{x^{\alpha}}
$$

formula (2.5) and the change of variables $\sigma \rightarrow T-\sigma$ show that both controls drive 0 to $\bar{y}_{\alpha}(x)$ in $x \geq T$. This can also be directly verified for $\bar{v}_{\alpha}(\sigma, x)$ :

$$
\int_{0}^{T} \bar{v}_{\alpha}(\sigma, x-(T-\sigma)) d \sigma=\frac{\sqrt{2 \alpha-1}}{x^{\alpha}} \int_{0}^{T} \sigma^{\alpha-1 / 2} d \sigma=\frac{\sqrt{2 \alpha-1}}{\alpha+1 / 2} \frac{T^{\alpha+1 / 2}}{x^{\alpha}} .
$$


The control $v(\sigma, x)$ is now defined by

$$
v(\sigma, x)= \begin{cases}\tilde{u}_{\alpha}(\sigma, x) & (\sigma, x) \in \mathcal{K} \\ \bar{u}_{\alpha}(\sigma, x) & (\sigma, x) \in \mathcal{C}_{1} \\ \bar{v}_{\alpha}(\sigma, x) & (\sigma, x) \in \mathcal{C}_{2}\end{cases}
$$

and we have

$$
\begin{aligned}
\|v(\sigma, \cdot)\|_{L^{2}(0, \infty)}=\int_{0}^{\infty} v(\sigma, x)^{2} d x \\
=\int_{0}^{\sigma} v(\sigma, x)^{2} d x+\int_{\sigma}^{\sigma+N} v(\sigma, x)^{2} d x+\int_{\sigma+N}^{\infty} v(\sigma, x)^{2} d x \\
\leq \frac{K(\alpha)}{K(3 / 2)} \int_{0}^{\sigma} \bar{u}_{\alpha}(\sigma, x)^{2} d x+\int_{\sigma}^{\sigma+N} \bar{u}_{\alpha}(\sigma, x)^{2} d x+\int_{\sigma+N}^{\infty} \bar{v}_{\alpha}(\sigma, x)^{2} d x \\
=\frac{K(\alpha)}{K(3 / 2)}\left(1-\left(\frac{T-\sigma}{T}\right)^{2 \alpha-1}\right)+\left(\left(\frac{T-\sigma}{T}\right)^{2 \alpha-1}-\left(\frac{T-\sigma}{T+N}\right)^{2 \alpha-1}\right) \\
\quad+\left(\frac{\sigma}{T+N}\right)^{2 \alpha-1}=\eta(\sigma)
\end{aligned}
$$

We have

$$
\begin{aligned}
\eta(0) & =1-\left(\frac{T}{T+N}\right)^{2 \alpha-1}<1 \\
\eta(T) & =\frac{K(\alpha)}{K(3 / 2)}+\left(\frac{T}{T+N}\right)^{2 \alpha-1}<1,
\end{aligned}
$$

the second inequality taking place for $N$ large enough. Since $\alpha \geq 3 / 2$ we have $2 \alpha-1 \geq 2$ and each of the three terms in (3.14) that make up $\eta(\sigma)$ have positive second derivative. It follows that $\eta(\sigma)$ is convex, thus (3.15) implies

$$
\eta(\sigma) \leq \max (\eta(0), \eta(T))<1
$$

and the control $v(\sigma, x)$ reaches the target $\bar{y}_{\alpha}(x)$ with smaller norm than $\bar{u}_{\alpha}(\sigma, x)$ thus proving that the latter is not norm optimal.

\section{Weakly singular controls, II}

We show in this section that the control $u_{3 / 2}(\sigma, x)$, although weakly singular, is time and norm optimal. To this end, we assume it not time optimal: then there exists an admissible control $u(\sigma, x)$ driving 0 to $\bar{y}_{3 / 2}(x)$ in time $T-\delta<T$. We show below that this implies that, for some $\alpha<3 / 2$ sufficiently close to $3 / 2$ the control $\bar{u}_{\alpha}(\sigma, x)$ is not norm optimal, which contradicts Theorem 3.2.

We construct a control $v(\sigma, x)$ that drives 0 to $\bar{y}_{\alpha}(x)$ in time $T$. This control is also constructed by pieces; the different domains are in Figure 6 .

Since $u(\sigma, x)$ drives 0 to $\bar{y}_{3 / 2}(x)$ in time $T-\delta$, the control

$$
\left\{\begin{array}{cc}
0 & (0 \leq \sigma<\delta) \\
u(\sigma-\delta, x) & (\delta \leq \sigma \leq T)
\end{array}\right.
$$




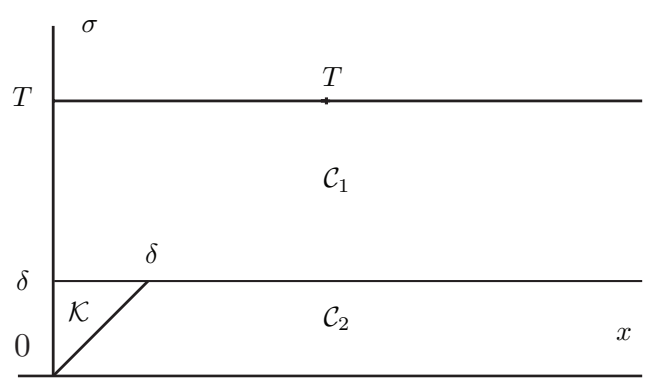

Figure 6

drives 0 to $\bar{y}_{3 / 2}(x)$ in time $T$. In view of (3.7), the control

$$
v_{1}(\sigma, x)= \begin{cases}0 & (0 \leq \sigma<\delta) \\ \frac{K(\alpha)}{K(3 / 2)} u(\sigma-\delta, x) & (\delta \leq \sigma \leq T)\end{cases}
$$

drives 0 to

$$
v_{1}(\sigma, x)= \begin{cases}\frac{K(\alpha)}{K(3 / 2)} \bar{y}_{3 / 2}(x)=\bar{y}_{\alpha}(x) & (0 \leq x \leq T) \\ \frac{K(\alpha) T^{2}}{y^{3 / 2}} & (T<x<\infty)\end{cases}
$$

in time $T$. We define next

$$
v_{2}(\sigma, x)= \begin{cases}\frac{K(\alpha)}{\delta}\left(\frac{T^{\alpha+1 / 2}}{(x+(T-\sigma))^{\alpha}}-\frac{T^{2}}{(x+(T-\sigma))^{3 / 2}}\right) & (\sigma, x) \in \mathcal{C}_{2} \\ 0 & (\sigma, x) \in \mathcal{K} \cup \mathcal{C}_{1} .\end{cases}
$$

This control drives to a target which is $=0$ in $0 \leq x \leq T$. Over the paths of integration $(\sigma, x-(T-\sigma))$ in formula $(2.5)$ for $x \geq T$ we have

$$
v_{2}(\sigma, x-(T-\sigma))= \begin{cases}\frac{K(\alpha)}{\delta}\left(\frac{T^{\alpha+1 / 2}}{x^{\alpha}}-\frac{T^{2}}{x^{3 / 2}}\right) & (0 \leq \sigma \leq \delta) \\ 0 & (\delta<\sigma<T)\end{cases}
$$

thus

$$
\int_{0}^{T} v_{2}(\sigma, x-(T-\sigma)) d \sigma=K(\alpha)\left(\frac{T^{\alpha+1 / 2}}{x^{\alpha}}-\frac{T^{2}}{x^{3 / 2}}\right) \quad(x \geq T)
$$

and it follows that $v_{2}(\sigma, x)$ drives to a "corrector" target $\bar{y}^{2}(x)$ such that $\bar{y}^{2}(x)=0$ in $0 \leq x \leq T$ and

$$
\bar{y}^{1}(x)+\bar{y}^{2}(x)=\bar{y}_{\alpha}(x) \quad(0 \leq x<\infty) .
$$

Accordingly, the control

$$
v(\sigma, x)=v_{1}(\sigma, x)+v_{2}(\sigma, x)
$$


drives 0 to the "right" target $\bar{y}_{\alpha}(x)$. It remains to select $\alpha$ so that $v(\sigma, x)$ does the drive with norm $<1$. On the one hand, we have

$$
\begin{aligned}
\|v(\sigma, \cdot)\|_{L^{2}(0, \infty)} & =\frac{K(\alpha)}{K(3 / 2)}\|u(\sigma-\delta, x)\|_{L^{2}(0, \infty)} \\
& \leq \frac{K(\alpha)}{K(3 / 2)}<1 \quad(\delta \leq \sigma \leq T)
\end{aligned}
$$

On the other hand, $\|v(\sigma, \cdot)\|_{L^{2}(0, \infty)}^{2}=\left\|v_{2}(\sigma, \cdot)\right\|_{L^{2}(0, \infty)}^{2}$ is constant in $0 \leq \sigma \leq \delta$ thus

$$
\begin{aligned}
& \|v(\sigma, \cdot)\|_{L^{2}(0, \infty)}^{2}=\left\|v_{2}(0, \cdot)\right\|_{L^{2}(0, \infty)}^{2} \\
& \quad=\frac{K(\alpha)^{2}}{\delta^{2}} \int_{0}^{\infty}\left(\frac{T^{\alpha+1 / 2}}{(x+T)^{\alpha}}-\frac{T^{2}}{(x+T)^{3 / 2}}\right)^{2} d x=\frac{K(\alpha)^{2}}{\delta^{2}} \int_{T}^{\infty}\left(\frac{T^{\alpha+1 / 2}}{x^{\alpha}}-\frac{T^{2}}{x^{3 / 2}}\right)^{2} \\
& \quad=\frac{K(\alpha)^{2}}{\delta^{2}}\left(T^{2 \alpha+1} \int_{T}^{\infty} x^{-2 \alpha} d x-2 T^{\alpha+5 / 2} \int_{T}^{\infty} x^{-\alpha-3 / 2} d x+T^{4} \int_{T}^{\infty} x^{-3} d x\right) \\
& \quad=\frac{K(\alpha)^{2} T^{2}}{\delta^{2}}\left(\frac{1}{2 \alpha-1}-\frac{2}{\alpha+1 / 2}+\frac{1}{2}\right) \\
& \quad=\frac{K(\alpha)^{2} T^{2}(2 \alpha-3)^{2}}{\delta^{2}\left(8 \alpha^{2}-2\right)}=L(\alpha) \rightarrow 0 \quad \text { as } \alpha \rightarrow \frac{3}{2} \quad(0 \leq \sigma \leq \delta)
\end{aligned}
$$

thus using (4.4) and (4.5) and taking $\alpha$ sufficiently close to $3 / 2$ in (4.5) we insure that

$$
\|v(\sigma, \cdot)\|_{L^{2}(0, \infty)} \leq L(\alpha)<1 \quad 0 \leq \sigma \leq \delta .
$$

In view of (4.4) and (4.6) we have constructed a control $v(\sigma, x)$ that drives 0 to $\bar{y}_{\alpha}(x)$ improving the norm of $\bar{u}_{\alpha}(\sigma, x)$. But $\bar{u}_{\alpha}(\sigma, x)$ is norm optimal by virtue of Theorem 3.2, thus a contradiction ensues and we are all done.

\section{Weakly singular controls, III}

The second counterexample involves the multiplier

$$
z(x)=\frac{e^{1 / 2 x}}{x} \quad(x>0) .
$$

We have

$$
\kappa(\sigma, z)^{2}=\int_{\sigma}^{\infty} \frac{e^{1 / x}}{x^{2}} d x=-\int_{\sigma}^{\infty}\left(e^{1 / x}\right)^{\prime} d x=e^{1 / \sigma}-1
$$

so that

$$
\kappa(\sigma, z)=\sqrt{e^{1 / \sigma}-1}=e^{1 / 2 \sigma} \sqrt{1-e^{-1 / \sigma}} .
$$

To estimate $\kappa(\sigma, z)$ near zero, we note that the positive function $f(\sigma)=e^{-1 / \sigma} / \sigma$ tends to zero for $\sigma \rightarrow 0, \sigma \rightarrow \infty$ and (since $f^{\prime}(\sigma)=e^{-1 / \sigma}(1-\sigma) / \sigma^{3}$ ) has a maximum at $\sigma=1$ where $f(1)=1 / e$. It follows that $e^{-1 / \sigma} \leq \sigma / e$ everywhere so that (giving up a lot)

$$
\kappa(\sigma, z)=e^{1 / 2 \sigma}\left(1+O\left(e^{-1 / \sigma}\right)\right)=e^{1 / 2 \sigma}(1+O(\sigma)) .
$$


This estimation shows that $\kappa(\sigma, z)$ is far from integrable in $[0, \infty)$, thus the function (5.1) is a multiplier in $\mathcal{Z}$ but it does not belong to $Z(T)$. We have

$$
\frac{1}{\kappa(\sigma, z)}=\frac{e^{-1 / 2 \sigma}}{1+O(\sigma)}=e^{-1 / 2 \sigma}(1+O(\sigma))
$$

for $\sigma$ near zero, thus

$$
\begin{aligned}
\omega(T, x, z) & =\int_{0}^{x} \frac{d \sigma}{\kappa(\sigma, z)}=\int_{0}^{x} e^{-1 / 2 \sigma} d \sigma+\int_{0}^{x} O(\sigma) e^{-1 / 2 \sigma} d \sigma \\
& =(1+O(x)) \int_{0}^{x} e^{-1 / 2 \sigma} d \sigma
\end{aligned}
$$

since

$$
\int_{0}^{x}|O(\sigma)| e^{-1 / 2 \sigma} d \sigma \leq C \int_{0}^{x} \sigma e^{-1 / 2 \sigma} d \sigma<C x \int_{0}^{x} e^{-1 / 2 \sigma} d \sigma
$$

Integrating by parts twice the last integral in (5.4) we obtain

$$
\begin{aligned}
\int_{0}^{x} e^{-1 / 2 \sigma} d \sigma & =2 \int_{0}^{x} \sigma^{2}\left(e^{-1 / 2 \sigma}\right)^{\prime} d \sigma=2 x^{2} e^{-1 / 2 x}-4 \int_{0}^{x} \sigma e^{-1 / 2 \sigma} d \sigma \\
& =2 x^{2} e^{-1 / 2 x}-8 \int_{0}^{x} \sigma^{3}\left(e^{-1 / 2 \sigma}\right)^{\prime} d \sigma \\
& =2 x^{2} e^{-1 / 2 x}-8 x^{3} e^{-1 / 2 x}+24 \int_{0}^{x} \sigma^{2} e^{-1 / 2 \sigma} d x .
\end{aligned}
$$

The function $g(\sigma)=\sigma^{2} e^{-1 / 2 \sigma}$ has derivative $g^{\prime}(\sigma)=e^{-1 / 2 \sigma}(2 \sigma+1 / 2)$ thus $g(\sigma)$ is increasing and we can estimate the last integral in (5.5) as follows:

$$
\int_{0}^{x} \sigma^{2} e^{-1 / 2 \sigma} d \sigma \leq x^{2} e^{-1 / 2 x} \int_{0}^{x} d \sigma=x^{3} e^{-1 / 2 x} .
$$

Putting together (5.4), (5.5) and (5.6) we deduce that the behavior of $\omega(T, x, z)$ near zero is described by

$$
\omega(T, x, z)=2 x^{2} e^{-1 / 2 x}(1+O(x)) .
$$

Lemma 5.1. The control

$$
\bar{u}(\sigma, x)=\frac{S(T-\sigma)^{*} z(x)}{\left\|S(T-\sigma)^{*} z(\cdot)\right\|}=\chi_{0}(x) \frac{z(x+(T-\sigma))}{\kappa(T-\sigma, z)} \quad(0 \leq t \leq T)
$$

associated with the multiplier (5.1) drives 0 to a target $\bar{y}(\cdot) \in D(A)$ in time $T$.

The proof of Lemma 5.1 requires checking that both $\bar{y}(\cdot)$ and $\bar{y}^{\prime}(\cdot)$ belong to $L^{2}(0, \infty)$. Observe first that $\kappa(x, z)$ is infinitely differentiable in $x>0$; since $\kappa(x, z) \neq 0$ the same is true of $1 / \kappa(x, z)$, and it follows from formula (2.10) that $\omega(T, x, z)$ is infinitely differentiable in $0<x \leq T$ and constant in $x \geq T$, the rightand left-sided derivatives different at $T$,

$$
\omega_{l}^{\prime}(T, T, z)=\frac{1}{\kappa(T, z)}=\frac{1}{\sqrt{e^{1 / T}-1}}, \quad \omega_{r}^{\prime}(T, T, z)=0 .
$$


From (5.7) and (5.1) we have

$$
\bar{y}(x)=\frac{e^{1 / 2 x}}{x} 2 x^{2} e^{-1 / 2 x}(1+O(x))=2 x(1+O(x))
$$

near $x=0$, thus $\bar{y}(x)$ is continuous in $x \geq 0$ and the boundary condition $\bar{y}(0)=0$ is satisfied. On the other hand

$$
\begin{aligned}
\bar{y}^{\prime}(x) & =z^{\prime}(x) \omega(T, x, z)+z(x) \omega^{\prime}(T, x, z)=z^{\prime}(x) \omega(T, x, z)+\frac{z(x)}{\kappa(x, z)} \\
& =\left(-\frac{e^{1 / 2 x}}{2 x^{3}}-\frac{e^{1 / 2 x}}{x^{2}}\right) 2 x^{2} e^{-1 / 2 x}(1+O(x))+\frac{e^{1 / 2 x}}{x} e^{-1 / 2 x}(1+O(x))
\end{aligned}
$$

where we have used the equality

$$
\omega^{\prime}(T, x, z)=\frac{1}{\kappa(x, z)} \quad(0<x<T)
$$

(consequence of (2.10)) and (5.3) to estimate $\omega^{\prime}(T, x, z)$. The bad terms cancel out and $\bar{y}(x)$ is continuously differentiable in $0 \leq x \leq T$, thus the proof of Lemma 5.1 is over.

We note that the fact that the target $\bar{y}(x)$ has a "corner" at $x=T$ is typical of targets for equation (2.4) (see for instance the graph of $\bar{y}_{\alpha}(x)$ in Figure 2).

Theorem 5.2. Let $T>0$ be arbitrary. Then the control $\bar{u}(\sigma, x)$ does not drive 0 to $\bar{y}(x)$ time or norm optimally.

Proof. Assume $\bar{u}(\sigma, x)$ drives 0 to $\bar{y}(x)$ time or norm optimally. Then, by Theorem $1.1 \bar{u}(\sigma, x)$ is regular, thus there exists a multiplier $\zeta(\cdot) \in Z(T)$ such that

$$
\bar{u}(\sigma, x)=\frac{S(T-\sigma)^{*} \zeta(x)}{\left\|S(T-\sigma)^{*} \zeta(\cdot)\right\|}=\chi_{0}(x) \frac{\zeta(x+(T-\sigma))}{\lambda(T-\sigma, z)} \quad(0 \leq t \leq T),
$$

where

and

$$
\lambda(x, \zeta)=\sqrt{\int_{x}^{\infty} \zeta(\sigma)^{2} d \sigma}
$$

$$
\int_{0}^{T} \lambda(x, z) d x<\infty
$$

Since $\bar{u}(\sigma, x)$ can be represented both by (5.5) and (5.8) we have

$$
\frac{\zeta(x+(T-\sigma))}{\lambda(T-\sigma, z)}=\frac{z(x+(T-\sigma))}{\kappa(T-\sigma, z)} \quad(0 \leq \sigma \leq T) .
$$

Over a characteristic $(\sigma, x-(T-\sigma))$ we have

which implies

$$
\frac{\zeta(x)}{\lambda(T-\sigma, z)}=\frac{z(x)}{\kappa(T-\sigma, z)} \quad(0 \leq \sigma \leq T),
$$

$$
\lambda(\sigma, z)=\frac{z(x)}{\zeta(x)} \kappa(\sigma, z)
$$


However, this is absurd in view of (5.9) and of the fact that

$$
\int_{0}^{T} \kappa(\sigma, z) d \sigma=\infty
$$

This ends the proof.

The multiplier (5.1) used in this example roughly corresponds to the multiplier used in [2], Section 5 for the semigroup (1.8) which satisfies $(a)\left\|S(T-t)^{*} z\right\|$ increases very fast as $t \rightarrow 0,(b) \bar{u}(t)$ drives 0 to a target $\bar{y} \in D(A)$. This example was elevated into a theorem in [3], Lemma 8.3 but the result is restricted to self-adjoint analytic semigroups, thus it cannot be applied to the right translation semigroup. It is remarkable that the present example, similar to the one in [2] works for the right translation semigroup.

The examples in this paper and [2] prompt the conjecture that growth (1.9) of the costate is the most that can be allowed for optimality irrespective of the semigroup $S(t)$; in other words, that a control associated with a costate that satisfies

$$
\lim _{t \rightarrow T}(T-t)\|z(t)\|_{E^{*}}=\lim _{t \rightarrow T}(T-t)\left\|S(T-t)^{*} z\right\|_{E^{*}}=\infty
$$

cannot be optimal. The evidence, of course, is insufficient to support this and it is not clear that the manipulations in [2] (much less those in this paper) could be twisted into proving a general result. It is also unknown whether there exist nonoptimal controls with associated costate satisfying (1.9).

\section{Adjoints}

It is worth noting that optimal problems for the system

$$
y^{\prime}(t)=A^{*} y(t)+u(t)
$$

with $A^{*}$ the adjoint of the operator $A$ in (2.3) behave in a totally different way than those for the system (1.1) The operator $A^{*}$ is given by

$$
A^{*} y(x)=y^{\prime}(x)
$$

with domain $D(A)=\left\{\right.$ all $y(\cdot) \in L^{2}(0, \infty)$ with $\left.y^{\prime}(\cdot) \in D(A)\right\}$ (no boundary conditions) The semigroup $S(t)^{*}$ generated by $A^{*}$ is the left translation semigroup (2.1) and satisfies (1.12) so that all multipliers $z^{*}(\cdot)$ belong to $L^{2}(0, \infty)$ and all norm or time optimal controls satisfy (1.5) with no conditions whatsoever on the target $\bar{y}^{*}(x)$. The semigroup $S(t)^{*}$ is associated with the control system

$$
\frac{\partial y(t, x)}{\partial t}=\frac{\partial y(t, x)}{\partial x}+u(t, x), \quad y(0, x)=\zeta(x), \quad(0 \leq t, x<\infty),
$$

in the sense that $S(t)$ is the propagation semigroup of the homogeneous equation $(u(t, x)=0)$. Since $\left(S(t)^{*}\right)^{*}=S(t)$ all time and norm optimal controls $\bar{u}(t)$ for this system satisfy

$$
\bar{u}(t)=\frac{S(T-t) z}{\|S(T-t) z\|}=\frac{S(T-t) z}{\|z\|},
$$


the second equality coming from the fact that $S(t)$ is isometric. This is also a sufficient condition for optimality. The first equality (2.2) implies that optimal trajectories starting at $\zeta$ are of the form

$$
\begin{aligned}
y^{*}(t) & =S(t)^{*} \zeta+\int_{0}^{t} S(t-\sigma)^{*} \bar{u}(\sigma) d \sigma \\
& =S(t)^{*} \zeta+\frac{1}{\|z\|} \int_{0}^{t} S(t-\sigma)^{*} S(T-\sigma) z d \sigma \\
& =S(t)^{*} \zeta+\frac{1}{\|z\|} \int_{0}^{t} S(t-\sigma)^{*} S(t-\sigma) S(T-t) z d \sigma \\
& =S(t)^{*} \zeta+\frac{S(T-t)}{\|z\|} \int_{0}^{t} z d \sigma=S(t)^{*} \zeta+\frac{t S(T-t) z}{\|z\|}
\end{aligned}
$$

and hit the target

$$
\bar{y}(T)=S(T) \zeta+\frac{T z}{\|z\|} .
$$

The control system (6.1) is essentially the only truly infinite dimensional example where "everything can be easily calculated". This is far from true for the control system (2.4) treated in this paper, whose only difference with (6.3) consists of the presence of a boundary condition.

\section{References}

[1] H. O. Fattorini, Time-optimal control of solutions of operational differential equations, SIAM J. Control 2 (1964) 54-59.

[2] H. O. Fattorini, Time optimality and the maximum principle in infinite dimension, Optimization 50 (2001) 361-385.

[3] H. O. Fattorini, A survey of the time optimal problem and the norm optimal problem in infinite dimension, Cubo Mat. Educational 3 (2001) 147-169.

[4] H. O. Fattorini, Existence of singular extremals and singular functionals, Jour. Evolution Equations 1 (2001) 325-347.

[5] H. O. Fattorini, Infinite Dimensional Linear Control Systems, North-Holland Mathematical Studies 201, Elsevier, Amsterdam 2005.

[6] H. O. Fattorini, Regular and strongly regular time and norm optimal controls, Cubo: A Mathematical Journal 10 (2008) 77-92.

H. O. Fattorini

University of California

Department of Mathematics

Los Angeles, California 90095-1555

USA

e-mail: hof@math.ucla.edu 